\title{
LES VARIATIONS DU RENDEMENT DE LA NOURRITURE CHEZ LES LARVES D'AESCHNIDAE EN FONCTION DE LEUR VITESSE DE CROISSANCE
}

\author{
[Odonates]
}

\author{
par P. Aguesse et P. Testakd
}

Le régime alimentaire des larves d'Odonates est connu depuis fort longtemps : il se compose uniquement de proies animales, de diverses tailles, capturées le plus souvent par projection rapide du masque lorsqu'elles viennent à passer à proximité de la larve qui se tient à l'affût.

Ces larves carnassières occupent ainsi, dans les milieux aquatiques où elles vivent, une place bien définie : elles utilisent la matière vivante élaborée par les herbivores aux dépens des végétaux et capturent parfois des organismes qui sont eux-mêmes des carnivores. C'est dire que les larves d'Odonates se situent, dans les chaînes alimentaires, à un niveau trophique élevé et que seuls les milieux riches, eutrophes, sont susceptibles de nourrir de nombreuses larves de Libellules tandis que les milieux oligotrophes ne permettent qu’à quelques larves d'effectuer la totalité de leur croissance. Le critère donc du nombre des individus présents dans un milieu aquatique constitue une indication précise de l'état trophique du milieu. C'est pour cette raison qu'il nous à semblé particulièrement intéressant d'étudier l'alimentation chez les larves d'Odonates en relation avec leur développement.

Dans une première approche, il est possible de considérer qu'en région tempérée les larves d'Odonates effectuent des croissances appartenant à deux types distincts. Certaines en effet se développent très rapidement : il ne s'écoule guère plus de 3 mois entre l'éclosion de l'œuf et la métamorphose imaginale. C'est le cas en particulier, outre les Lestes, d'Aeschna mixta et d'Anax parthenope chez les Aeschnidae - tout au moins en région méditerranéenne -, de différents Sympetrum et de Crocothemis erythraea chez les Libellulidae. D'autres espèces, par contre, exigent un an, ou deux ou trois, pour passer du stade ouf au stade imaginal : dans ce second cas, il y a succession de périodes de croissance rapide (pendant la belle 
saison) et de périodes de vie ralentie durant les mois d'hiver. C'est le cas le plus fréquent chez les Odonates et en particulier, chez les Aeschnidae, pour Aeschna cyanea, Anax imperator et Anax parthenope dans la région parisienne.

Le problème est en fait plus complexe car une même espèce dans une même région peut présenter les deux types de croissance larvaire : ainsi, certaines larves d'Aeschna cyanea provenant de la région parisienne peuvent-elles avoir une croissance rapide tandis que d'autres provenant de la même localité (on pourrait même dire provenant d'œufs pondus par la même femelle, car Schaller en 1962 a démontré que la vitesse de croissance et le nombre des mues larvaires sont fixés dès l'embryogenèse et que la descendance d'une unique femelle est, de ce point de vue, hétérogène) ont une croissance lente. A la température de $20^{\circ}$, dans les conditions de photopériode naturelle et avec un excès de nourriture toujours disponible, les larves à croissance rapide demandent moins de 3 mois pour effectuer leurs trois dernières mues larvaires, celles à croissance lente exigeant plus de huit mois pour effectuer cette même partic de leur croissance dans les mêmes conditions de milieu.

Quoi qu'il en soit, nous ne considérerons ici que deux exemples illustrant les deux types de développement larvaire : les conditions expérimentales d'élevage sont les mêmes dans les deux cas, ce qui facilite la comparaison des résultats obtenus.

\section{L Larves à croissance rapide.}

Un lot d'une douzaine de larves d'Anax parthenope récolté dans un étang de Camargue à la fin d'avril 1964 fut élevé en laboratoire à une température constante de $20^{\circ}$ et soumis à la photopériode naturelle. Chaque larve récoltée pesait aux environs de $10 \mathrm{mg}$; après 6 mues, elles parvenaient au dernier stade larvaire et pesaient alors aux environs de $1300 \mathrm{mg}$. Elles se métamorphosaient dès la mi-août : ainsi, en trois mois et demi, ces larves ont élaboré plus d'un gramme de matière vivante. Leurs poids aux différents stades de leur croissance furent les suivants* :

\begin{tabular}{|c|c|c|c|c|c|c|c|}
\hline $\begin{array}{c}\text { Stades } \\
\text { Poids }\end{array}$ & 6 & $\frac{7}{6}$ & $\frac{8}{10}$ & $\frac{11}{10}$ & 12 \\
\hline (en mg) & $10-21$ & $32-62$ & $79-143$ & $143-277$ & $282-575$ & $590-1035$ & $1031-1324$ \\
\hline
\end{tabular}

* Nous considérons chez les Aeschnidae le stade pré-imaginal comme le douzième, ce qui est exact pour de nombreuses espèces mais ne semble pas l'être chez Anax parthenope qui n'effectuerait que 10 mues larvaires. Toutefois, pour homogénéiser les résultats et faciliter les comparaisons avec Aeschna cyanea en particulier, nous considérons que les derniers stades de la vic larvaire d'A. parthenope sont les stades 6 à 12 . 
Dans ce tableau, les valeurs indiquées pour chacun des stades correspondent aux poids au début et à la fin du stade considéré : c'est dire qu'au cours de chacun des stades les larves d'Anax parthenope à croissance rapide doublent approximativement leur poids, à l'exception du dernier stade larvaire, celui pendant lequel se prépare la métamorphose.

La consommation de nourriture au cours de la croissance larvaire de cette espèce a été régulièrement notée pour les 5 derniers stades. Nous donnons ci-après les valeurs moyennes : observons toutefois qu'il n'y a, pour cette espèce, que peu d'écarts par rapport à ces valeurs, et que cette homogénéité relative de la croissance et de la consommation de la nourriture paraît bien être une caractéristique des espèces à croissance rapide.

\begin{tabular}{|c|c|c|c|}
\hline Stades & $\begin{array}{c}\text { Nourriture } \\
\text { consommée } \\
\text { (en mg) }\end{array}$ & $\begin{array}{c}\text { Durée du stade } \\
\text { (en jours) }\end{array}$ & $\begin{array}{c}\text { Consommation } \\
\text { journalière } \\
\text { (en mg) }\end{array}$ \\
\hline 8 & 174 & 12,4 & 14 \\
$\mathbf{9}$ & $\mathbf{3 2 7}$ & 16,5 & 19,7 \\
$\mathbf{1 0}$ & $\mathbf{8 3 9}$ & 22,5 & $\mathbf{3 7 , 2}$ \\
$\mathbf{1 1}$ & 1659 & 27,2 & $\mathbf{6 0 , 8}$ \\
$\mathbf{1 2}$ & $\mathbf{3 4 1 3}$ & 36,3 & $\mathbf{9 4}$ \\
\hline
\end{tabular}

La nourriture consommée globalement au cours de chacun des cinq derniers stades larvaires double en valeur pondérale, tout comme double le poids de chaque larve. Cependant, si nous tenons compte de la durée de chaque stade et que nous considérons la consommation journalière et non plus globale, nous nous rendons compte que celle-ci n'augmente que des deux tiers environ d'un stade au stade suivant. Il est aussi évident en considérant la consommation journalière de nourriture que son augmentation est moins importante au cours du $12^{\circ}$ stade qu'au cours des stades précédents : ceci nous conduit à examiner la production de matière vivante au cours de la croissance larvaire et sa répartition dans le temps, puis à envisager le rendement de la nourriture consommée par les larves d'Anax parthenope.

En moyenne, pour chacun des stades 8 à 12 dont la durée a été indiquée dans le tableau précédent, la production de matière vivante a été la suivante :

\begin{tabular}{|c|c|c|}
\hline Stades & $\begin{array}{c}\text { Production globale } \\
\text { pour le stade (en mg) }\end{array}$ & $\begin{array}{c}\text { Production journalière } \\
\text { (en mg) }\end{array}$ \\
\cline { 1 - 2 } 8 & 68,8 & 5,5 \\
9 & 134,4 & 8,1 \\
10 & 296,1 & 13,1 \\
11 & 445 & 16,3 \\
12 & 292,5 & 8,0 \\
\hline
\end{tabular}


Nous observons donc un accroissement régulier de la production journalière jusqu'au onzième stade inclus, puis une brusque diminution de cette production au cours du $12^{\circ}$ stade. Il faut donc s'attendre à ce que le rendement de la nourriture absorbée diminue également à la fin de la vie larvaire, ce rendement étant défini comme le rapport de la matière vivante produite à la nourriture assimilée.

\begin{tabular}{|r|c|}
\hline Stades & Rendements moyens \\
\cline { 2 - 2 } $\mathbf{8}$ & 0,39 \\
9 & 0,41 \\
10 & 0,35 \\
11 & 0,26 \\
12 & 0,085 \\
\hline
\end{tabular}

Le rendement diminue effectivement de façon fort sensible au cours du dernier stade de la vie larvaire d'Anax parthenope, mais nous observons en outre qu'il atteint des valeurs comparables pour les stades 8,9 et 10 et qu'il est déjà en diminution au cours du onzième stade. A ce stade, les larves d'Anax parthenope pèsent entre 590 et $1035 \mathrm{mg}$ et une partie importante de la nourriture assimilée est utilisée pour l'entretien : ainsi, nous pouvons noter au cours de la croissance larvaire, une progression de la ration d'entretien au détriment de la fraction utilisée pour la production de matière vivante, et cette progression devient tout à fait évidente à partir du onzième stade. Au cours du douzième stade, le phénomène s'accentue et devient parfaitement net : la nourriture consommée (3413 mg) est plus du double de celle consommée pendant le stade précédent (1 $659 \mathrm{mg}$ ), mais la matière animale produite n'atteint qu'une valeur sensiblement égale aux deux tiers de celle produite au cours du onzième stade (292 $\mathrm{mg}$ contre $445 \mathrm{mg}$ ). Du point de vue écologique, cette observation est importante car la place occupée par les larves d'Aeschnidae dans les chaînes trophiques varie, de façon quantitative, au cours de l'existence des larves. De plus, ces Insectes sont d'excellents transformateurs d'énergie pendant la majeure partie de leur vie larvaire, mais au moment où, pondéralement, ils tiennent une place importante dans le milieu leur rendement énergétique diminue dans des proportions telles qu'ils ne sont plus que de mauvais transformateurs d'énergie. Ils consomment de l'énergie sans production importante, se comportant alors en « profiteurs » des chaînes trophiques.

\section{II. - Larves à croissance lente.}

Plusieurs lots de larves d'A eschna cyanea furent mis en expérience qui tous avaient pour origine une unique localité de la région parisienne. Placées dans les mêmes conditions expérimentales que 
celles d'Anax parthenope, certaines firent une croissance continue et rapide tandis que d'autres firent une croissance lente. Que leur croissance fût lente ou rapide, les larves d'A eschna cyanea eurent des poids tout à fait comparables pour les trois derniers stades, et nous indiquerons la moyenne notée pour chaque stade sans tenir compte de la vitesse de croissance :

\begin{tabular}{|c|c|c|c|}
\hline Stades & 10 & 11 & $\frac{12}{250-1147}$ \\
\hline poids (en mg) & $\frac{255-509}{467-899}$ & $\mathbf{8 8 1 - 1 1 4 7}$ \\
\hline
\end{tabular}

Ces poids sont, en moyenne, inférieurs à ceux notés pour ces mêmes stades chez Anax parthenope et la différence s'accentue du dixième au douxième stade, passant de $30 \mathrm{mg}$ environ à plus de $160 \mathrm{mg}$ à la fin du dernier stade larvaire. Notons encore que chez les Aeschna cyanea il y a sensiblement doublement du poids entre le début et la fin de chaque stade, quelle que soit la vitesse de croissance.

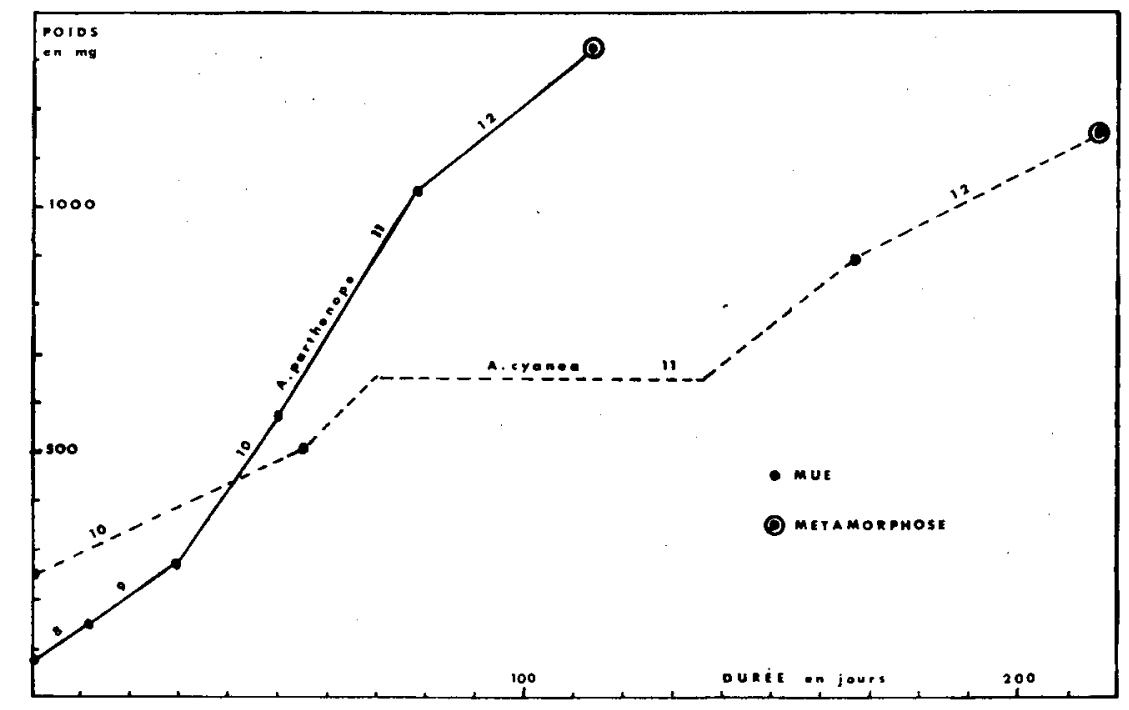

Fig. 1 : Croissance pondérale des larves d'Anax parthenope et d'Aeschna cyanea; 8 à 12 : stades larvaires.

La nourriture consommée durant les trois derniers stades larvaires d'A. cyanea (celles ayant effectué une croissance lente) se présente 
de façon sensiblement différente de ce que nous avions constaté pour Anax parthenope :

\begin{tabular}{|c|c|c|c|}
\hline Stades & $\begin{array}{c}\text { Nourriture } \\
\text { consommée } \\
\text { (en mg) }\end{array}$ & $\begin{array}{c}\text { Durée du stade } \\
\text { (en jours) }\end{array}$ & $\begin{array}{c}\text { Consommation } \\
\text { journalière } \\
\text { (en mg) }\end{array}$ \\
\cline { 1 - 3 } 10 & 1523 & 56,3 & 27 \\
11 & 2530 & 112,5 & 22,5 \\
12 & 2328 & 50 & 46,5 \\
\hline
\end{tabular}

Nous ne notons plus ici une progression régulière de la consommation journalière : au contraire, celle-ci est nettement moins élevée au cours du $11^{\circ}$ stade qu'au cours du stade précédent. Ceci s'explique par le fait que ce onzième stade fut un stade de repos pendant lequel la croissance pondérale n'eut lieu qu'à proximité

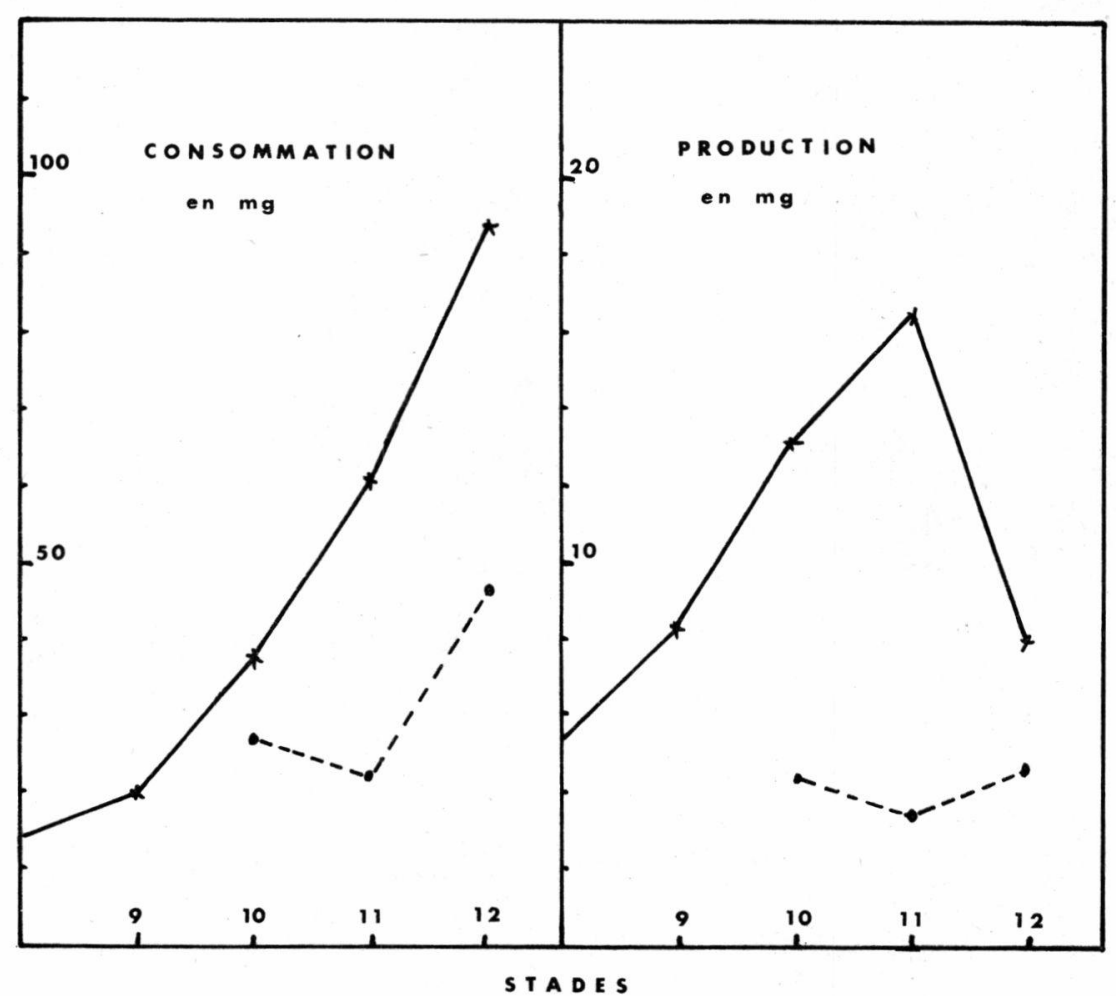

FIG. 2 : Consommation et production journalières chez les larves d'Anax parthenope et d'Aeschna cyanea au cours des derniers stades larvaires; $A$. parthenope en trait plein, $A$. cyanea en tirets. 
des mues du début et de la fin du stade. Pendant plus de deux mois, les larves restèrent presque totalement inactives, se nourrissant seulement de temps à autre : durant ce laps de temps, elles maintinrent leur poids sans l'augmenter ni le diminuer et l'on peut considérer que la nourriture absorbée au cours de cette longue période correspond approximativement à la ration d'entretien des larves d'Aeschna cyanea à la température de $20^{\circ}$. Cette ration d'entretien atteint une valeur journalière comprise entre 10 et $13 \mathrm{mg}$. Si les résultats obtenus pour la consommation de nourriture pendant la périodes d'inactivité des larves sont relativement homogènes, il n'en est pas de même de ceux obtenus pour les périodes actives et les écarts notés par rapport aux valeurs moyennes citées dans le tableau ci-dessus sont souvent importants. Rappelons qu'au contraire pour Anax parthenope les écarts par rapport aux moyennes étaient négligeables.

La production de matière vivante, chez les larves à croissance lente, est aussi très différente de celle observée pour les larves à croissance rapide et nous remarquerons immédiatement la valeur très faible de la production au cours du onzième stade caractérisé par une longue période d'inactivité.

\begin{tabular}{|c|c|c|}
\hline Stades & $\begin{array}{c}\text { Croissance globale } \\
\text { pour le stade (en mg) }\end{array}$ & $\begin{array}{c}\text { Production journalière } \\
\text { (en } \mathrm{mg})\end{array}$ \\
\hline 10 & 244 & 4,3 \\
11 & 395 & 3,5 \\
12 & 232 & 4,6 \\
\hline
\end{tabular}

Toutes les valeurs sont ici comprises entre 3 et 5 mg par jour, tandis que pour $A$. parthenope elles étaient toujours supérieures à $5 \mathrm{mg}$ et comprises entre 5 et $17 \mathrm{mg}$ par jour. Notons que les larves d'A. cyanea qui effectuent une croissance rapide ont des valeurs de production journalière qui sont tout à fait comparables à celles observées pour A. parthenope.

Les rendements chez les larves à croissance lente sont aussi très faibles en comparaison de ceux notés pour les larves à croissance rapide.

\begin{tabular}{|c|c|}
\hline Stades & Rendements moyens \\
\hline 10 & 0,16 \\
11 & 0,15 \\
12 & 0,10 \\
\hline
\end{tabular}


Chez Anax parthenope, pour ces mêmes stades, ils étaient respectivement de $0,35,0,26$ et 0,085 . C'est dire que seul le rendement du douzième stade chez les larves à croissance rapide est comparable au rendement observé pendant toute la croissance larvaire des espèces à développement lent. Ainsi, pendant la majeure partie de l'année, les larves d'Aeschnidae à croissance lente consomment des quantités importantes de nourriture et ne produisent que peu de matière vivante tandis que les larves à croissance rapide consomment des quantités de nourriture comparables mais produisent de la matière vivante en quantité nettement supérieure pendant la majeure partie de leur vie larvaire.

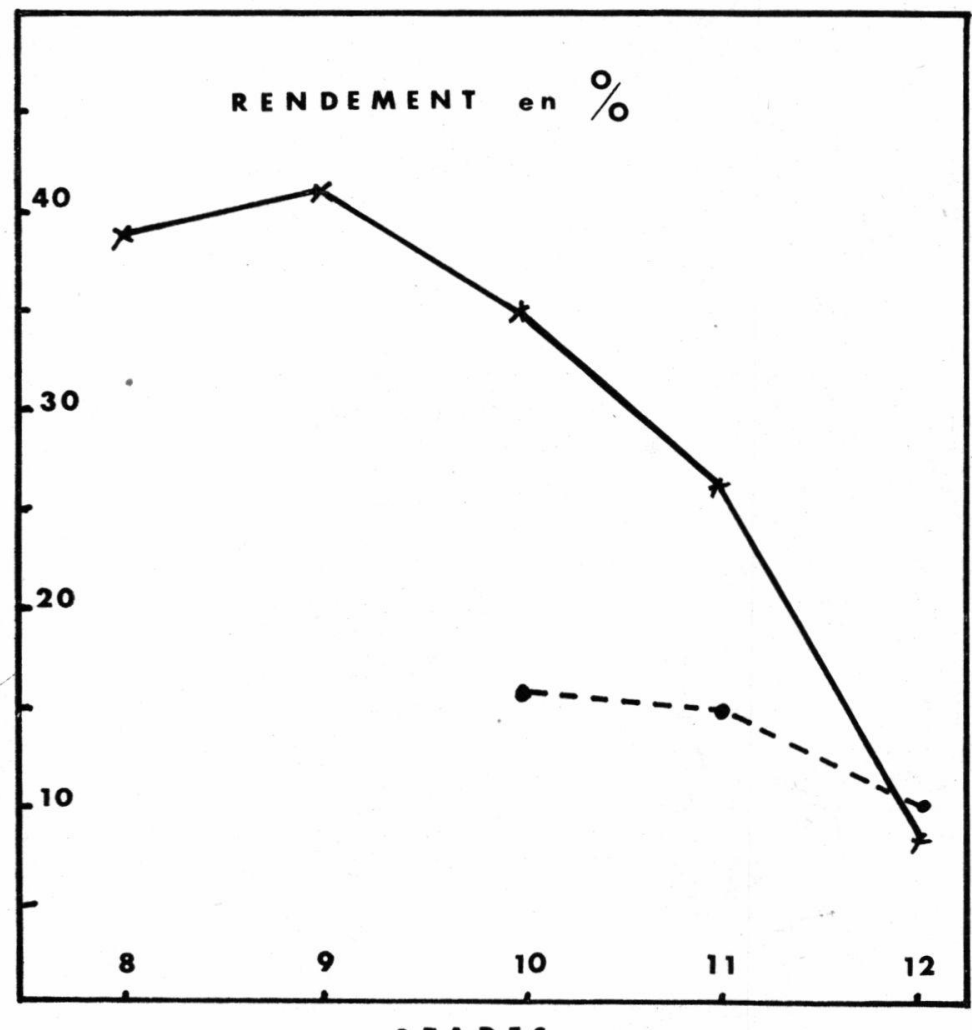

S TA DES

FIG. 3 : Evolution des rendements chez les larves d'Anax parthenope et d'Aeschna cyanea au cours des derniers stades larvaires; A. parthenope en trait plein, $A$. cyanea en tirets. 


\section{Conclusions}

Cette différence importante dans le métabolisme larvaire chez les Odonates a une influence notable sur l'énergétique du peuplement d'un milieu aquatique : les larves à croissance rapide mettent rapidement à la disposition de leurs prédateurs une quantité appréciable de matière vivante tandis que les larves à croissance lente, partant de la même quantité globale de nourriture disponible, ne mettent que dans un laps de temps beaucoup plus long la même quantité de matière vivante à la disposition de leurs prédateurs. Il est certain qu'il y a, outre la différence d'intensité dans le métabolisme, une différence dans la répartition dans le temps de la nourriture nécessaire à la production de matière vivante : lorsque la croissance est rapide, les larves doivent trouver en peu de temps une nourriture abondante; par contre, lorsque la croissance est lente, elles doivent trouver pendant une longue période une quantité de nourriture moins importante à chaque instant, mais globalement égale à celle nécessaire à la croissance rapide. C'est en relation avec cette observation qu'il faut remarquer que les Odonates dont les larves effectuent une croissance rapide se trouvent dans les milieux eutrophes où les pullulations passagères d'organismes sont fréquentes, tandis que les larves des espèces à croissance lente colonisent le plus souvent des milieux oligotrophes dans lesquels elles trouvent régulièrement toute l'année une petite quantité de nourriture. Ainsi les milieux eutrophes sont-ils caractérisés non seulement par l'abondance et la richesse en matière vivante, mais aussi par la rapidité et le haut rendement du métabolisme énergétique, tandis que les milieux oligotrophes sont caractérisés autant par leur pauvreté relative en matière vivante que par la lenteur et le faible rendement du métabolisme énergétique.

Il est bien évident que ces observations doivent être vraies à tous les niveaux trophiques d'un même milieu, faute de quoi les différents maillons d'une chaîne trophique ne pourraient trouver une nourriture suffisante. Autrement dit, un métabolisme rapide et à haut rendement au niveau de carnivores tels que les larves d'Odonates implique obligatoirement un métabolisme rapide et à haut rendement au niveau des herbivores ainsi qu'une grande abondance et un taux de renouvellement élevé au niveau des producteurs primaires, en particulier ai niveau du phytoplancton.

(Laboratoire de Zoologie, Faculté des Sciences, 45 - Orléans-La Source.

Laboratoire de Zoologie, E.N.S., 24, rue Lhomond, 75 - Paris $\left(5^{\circ}\right)$. 


\section{RÉSUMÉ}

En prenant leurs exemples chez les larves d'Aeschnidae, les auteurs mettent en évidence chez les Odonates une croissance rapide d'une part, caractérisée par un rendement élevé, et une croissance lente d'autre part, caractérisée par un faible rendement. Les auteurs pensent qu'un métabolisme élevé est nécessaire à tous les niveaux d'une chaîne trophique qui se termine par des carnivores à haut rendement et qu'ainsi les caractères d'eutrophie et d'oligotrophie des eaux doivent correspondre autant à une quantité globale de matière vivante qu'à une intensité du métabolisme.

\section{VARIATIONS IN THE FOOD EFFICIENCY \\ OF THE AESCHNIDAE LARVAE \\ IN RELATION TO THEIR RATE OF GROWTH}

Taking their examples from the Aeschnidae larvae, the authors point out in the dragonfly a rapid growth, on one hand, characterized by a heightened efficiency and a slow growth, on the other hand, characterized by a lowered efficiency. The auhors think that a heightened metabolism is necessary at all levels of the trophic chain, which ends with the highly productive carnivores and therefore that the characteristics of eutrophy and oligotrophy of the waters must correspond as much to the total quantity of living matter as to the intensity of the metabolism.

\section{DIE VARIATIONEN DER RENTABILTTAT DER ERNÄHRUNG BEI DEN LARVEN AESCHNIDAE UNTER BERUCKSICHTIGUNG IHRES SCHNELLEN WACHSTUMS}

Indem die Autoren die Larven Aeschnidae als Beispiel heranziehen, stellen sie bei den Odonata einerseits ein schnelles Wachstum heraus, das durch eine starke Rentabilität charakterisiert ist, andererseits ein langsamer Wachstum, charakterisiert durch eine schwache Rentabilität. Die Autoren glauben, dass ein hoher Metabolismus in allen Stufen der Nahrungsfolge notwendig ist, der bei den Karnivoren mit hoher Rentabilität gipfelt. So müssen die Eigenschaften der Eutrophie und der Oligotrophie des Wassers sowohl der Gesamtmenge einer lebendigen Materie als auch der Intensität des Metabolismus entsprechen.

\section{BIBLIOGRAPHIE}

Aguesse (P.). 1968. - Les Odonates, in Faune de l'Europe Occidentale, 4, Masson, Paris, 258 pp.

Aguesse (P.) et Testard (P.). 1967. - Développement et productivité des larves d'Aschnidae, in Lamotte et Bourlière, Problèmes de productivité biologique, Masson, Paris : 183-198.

Schaller (F.). 1962. - Rôle de la diapause et de la vitesse du développement dans le cycle biologique d'Aeschna cyanea. Bull. Ass. Phil. Alsace-Lorraine, 11, 3-4 : 128-137.

Testard (P.). 1968. — Développement et productivité des larves d'Aeschnidae. Thèse troisième cycle. Paris : $51 \mathrm{pp}$. 